\title{
A Novel Routing Protocol based on Context Information on Successful Message Delivery and Buffer Overflow in Opportunistic Delay Tolerant Networks
}

\author{
Sun Woong $\mathrm{Kim}^{1}$, Dong Young $\mathrm{Seo}^{2}$ and Yun Won Chung ${ }^{1 *}$ \\ ${ }^{1}$ School of Electronic Engineering, Soongsil University, Seoul, Korea \\ ${ }^{2}$ Department of Information and Telecommunication Engineering, Graduate \\ School, Soongsil University, Seoul, Korea \\ E-mail: \{gjgjgjgj, seodong2da, ywchung\}@ssu.ac.kr
}

\begin{abstract}
In delay opportunistic delay tolerant networks, message is delivered to a destination node by a store-carry-forward mechanism. In this paper, a novel routing protocol using contact information on successful message delivery and buffer overflow is proposed in opportunistic delay tolerant networks, where a message is selected to be forwarded to another node with lower difference between the number of dropped messages and the number of successfully delivered messages as well as higher delivery predictability for the considered message. Then, selected messages are transmitted based on the ascending order of hop count firstly and the descending order of delivery predictability of the receiving node secondly when the hop counts are equal. The performance of the proposed protocol is analyzed in detail, from the aspect of delivery ratio, overhead ratio, and delivery latency, by varying the buffer size, message interval, and number of nodes. Numerical results show that the proposed protocol with the proposed transmission priority has the best performance.
\end{abstract}

Keywords: routing protocol, contact information, opportunistic delay tolerant networks

\section{Introduction}

In delay tolerant network (DTN), a message is delivered to a destination node from a source node based on opportunistic routing protocol, since there is no guaranteed end-toend routing path, and most of the connections between nodes are temporary. Example scenarios for applying DTN include disaster environment, where infrastructure is destroyed and thus, communication should be carried out using sparsely distributed peer nodes. In DTN, when a node generates a message, it stores the message in its buffer and carries it while moving. Then, if it contacts another node opportunistically, it forwards the message to another node, if forwarding condition is satisfied. This kind of message delivery mechanism is called 'store-carry-forward' mechanism [1-3].

One of the representative opportunistic routing protocol for DTN is probabilistic routing protocol for intermittently connected networks (PRoPHET), which was standardized in IETF [4]. In PRoPHET, delivery predictability between two nodes, which is defined as a value between 0 and 1 , is calculated based on contact history between them. The delivery predictability between any two nodes has its initial value and it is increased whenever they contact each other, and the delivery predictability decreases while there is no contact between them. Therefore, the value of delivery predictability varies depending on contact between two nodes and can be used to determine which node is more

* Corresponding Author 
preferable to forward a message to a destination node. Therefore, PRoPHET protocol is better than Epidemic protocol by efficiently controlling the number of message copies and forwarding to more probable nodes to deliver messages to destinations [5].

Many works on improving the performance of the PRoPHET protocol have been carried out recently [6]-[13]. In [6], message forwarding is restricted efficiently using sociality of nodes. In [7], message forwarding is restricted effectively based on the number of message copies. In [8], either Epidemic or PRoPHET is selected dynamically based on the density of simultaneous contact nodes. In [9], contact duration as well as contact frequency is considered for message forwarding. In PRoPHET+ [10], a new metric of deliverability is defined and a message is forwarded if the deliverability of receiving node is higher than that of the sending node, where deliverability is defined as a weighted sum of battery power, location, buffer size, popularity, and delivery predictability. In [11], a node checks if the delivery predictability of another node is higher than minimum threshold value and the sum of delivery predictabilities of forwarded nodes for the considered message is smaller than maximum threshold value. In [12], if a message is delivered to a destination node successfully, an acknowledgment messages are generated at the destination node and the last forwarding node. Then, a node with the considered message deletes it if it contacts another node with the acknowledgment of the considered message. In [13], the number of successfully delivered messages as well as delivery predictability is considered to improve the performance of the PRoPHET protocol.

In PRoPHET protocol, however, it is still necessary to limit the number of message forwarding more efficiently since buffer may overflow due to large number of forwarding, if the number of messages generated is high. To improve the performance of the conventional PRoPHET protocol, in this paper, message drop count and success count are recorded, and count difference is newly defined as the subtraction of success count from drop count, which was proposed in our preliminary work [14]. Then, a novel opportunistic routing protocol is proposed, where a message is selected to be forwarded to another node with lower count difference as well as higher delivery predictability for the considered message. Finally, messages are transmitted with priority, based on the hop count and delivery predictability of the receiving node. As an extension of our preliminary work in [14], the proposed work is different significantly from the previous work from the following aspects:

In our preliminary work, messages which should be forwarded are selected based on count difference and delivery predictability but no priority between selected messages was assumed when transmitting them to a contact node. In this work, however, selected messages are transmitted with priority, based on the ascending order of hop count firstly and the descending order of delivery predictability of the receiving node secondly when the hop counts are equal.

In our preliminary work, only the idea of forwarding scheme was presented without any performance validation. In this work, however, the performance of the proposed protocol is analyzed extensively, based on the simulation using ONE simulator from the aspect of delivery probability, overhead ratio, and delivery latency. Then, we show that the proposed protocol performs better than the PRoPHET protocol by varying the buffer size, message generation interval, and number of nodes.

The remainder of this paper is organized as follows: In Section 2, the proposed protocol is illustrated using examples and a detailed algorithm of the proposed protocol is proposed using flowchart. In Section 3, the performance of the proposed protocol is analyzed extensively using simulation. Finally, Section 4 summarizes this work. 


\section{The Proposed Protocol}

In the proposed protocol, we count the number of dropped message due to buffer overflow and it is defined as 'drop count' in this paper. Also, we count the number of successfully delivered messages by the node and it is defined as 'success count'. Then, 'count difference' is defined as follows:

Count difference $=$ drop count - success count

Figure 1 shows an example of drop count and success count updates. When node A and node $\mathrm{B}$ contact, node $\mathrm{A}$ delivers message $\mathrm{m} 2$ to node $\mathrm{B}$ since node $\mathrm{B}$ is the destination node of message $\mathrm{m} 2$. Then, success count of node A increases by one. Since the delivery predictability of node $\mathrm{B}$ to node $\mathrm{F}$ for message $\mathrm{m} 7$ is higher than that of node $\mathrm{A}$ to node $\mathrm{F}$, the message $\mathrm{m} 7$ should be forwarded to node B. Also, since the delivery predictability of node $\mathrm{B}$ to node $\mathrm{G}$ for message $\mathrm{m} 5$ is higher than that of node $\mathrm{A}$ to node $\mathrm{G}$, the message $\mathrm{m} 5$ should be forwarded to node $\mathrm{B}$. In the example, it is assumed that the available buffer space of node B is not sufficient to accommodate $\mathrm{m} 5$ and $\mathrm{m} 7$, and only one message can be accommodated. Then node $\mathrm{B}$, in order to accommodate $\mathrm{m} 5$ and $\mathrm{m} 7$, should remove one message in its buffer, which is stored in node B's buffer for the longest time, and the drop count of node B increases by 1 .

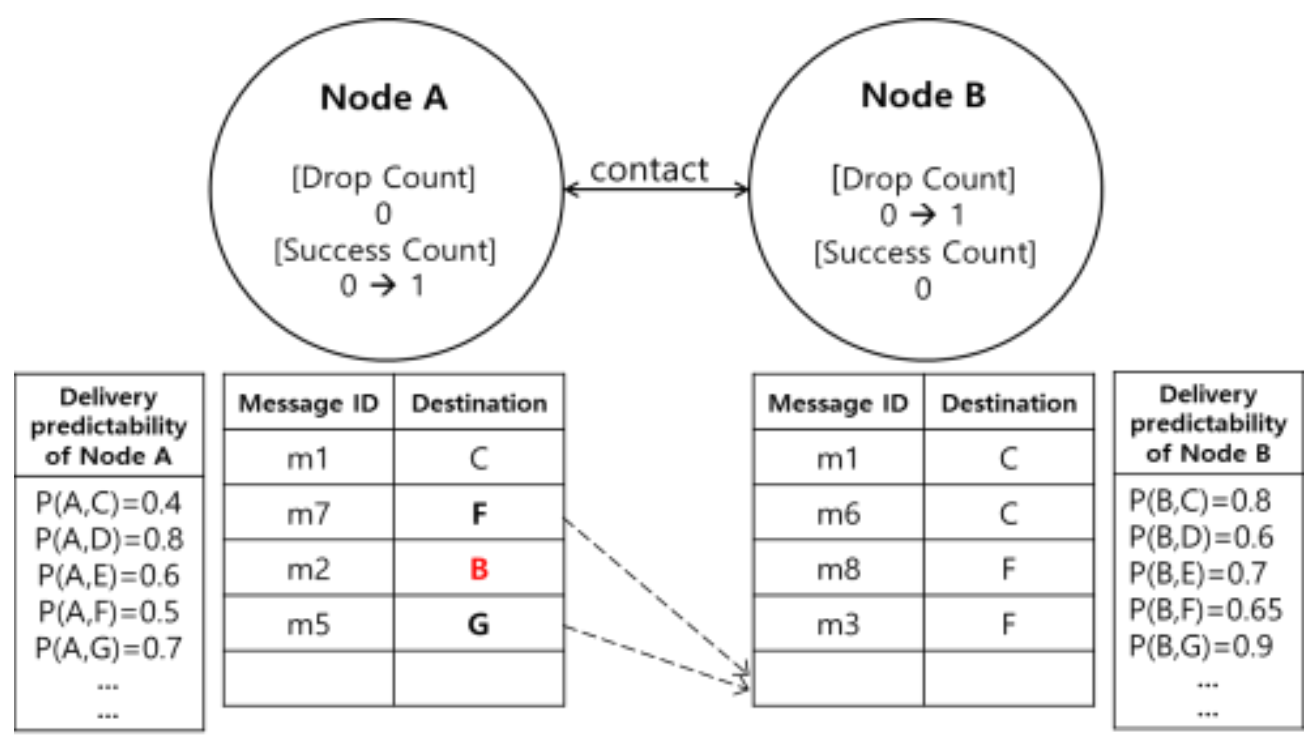

Figure 1. Example of Drop Count and Success Count Update

Figure 2 shows a detailed flowchart of the proposed protocol. In the proposed protocol, each node updates hop count, drop count, success count, and count difference. When node A and node B contacts they firstly exchange summary vectors. If node A has message which does not exist in node B's buffer, it checks if node B is the destination node of a message of the sending node A. If it is, the message is forwarded to node B, and the success count of the node $\mathrm{A}$ increases by 1 . If the destination node of the message is not node $\mathrm{B}$, node $\mathrm{A}$ firstly checks the proposed criteria in order to decide whether the message should be forwarded to node B or not. In the proposed protocol, if a message is generated by a node originally and has not been forwarded to another node yet, the hop count of the message is defined as 0 . Afterwards, whenever the message is forwarded to another node, the hop count of the message increases by 1 . If the hop count of the considered message is 0 , the message is selected to be forwarded to node $\mathrm{B}$, only if node $\mathrm{B}$ has either lower count difference or higher delivery predictability in order not to limit the dissemination of the message unnecessarily. If hop count of the considered message is not 0 , the message is selected to be forwarded to node B if node B has both lower count 
difference and higher delivery predictability for this message. By doing this, a node with more drop counts is avoided as forwarding node in order to reduce message drop and increase delivery ratio of messages. If all the messages are checked, node A sorts messages in the ascending order of hop count firstly and messages are transmitted based on the sorted order. If there are messages with the same hop count, then the messages are sorted based on the descending order of delivery predictability of the receiving node. Then, the messages are transmitted based on the sorted order.

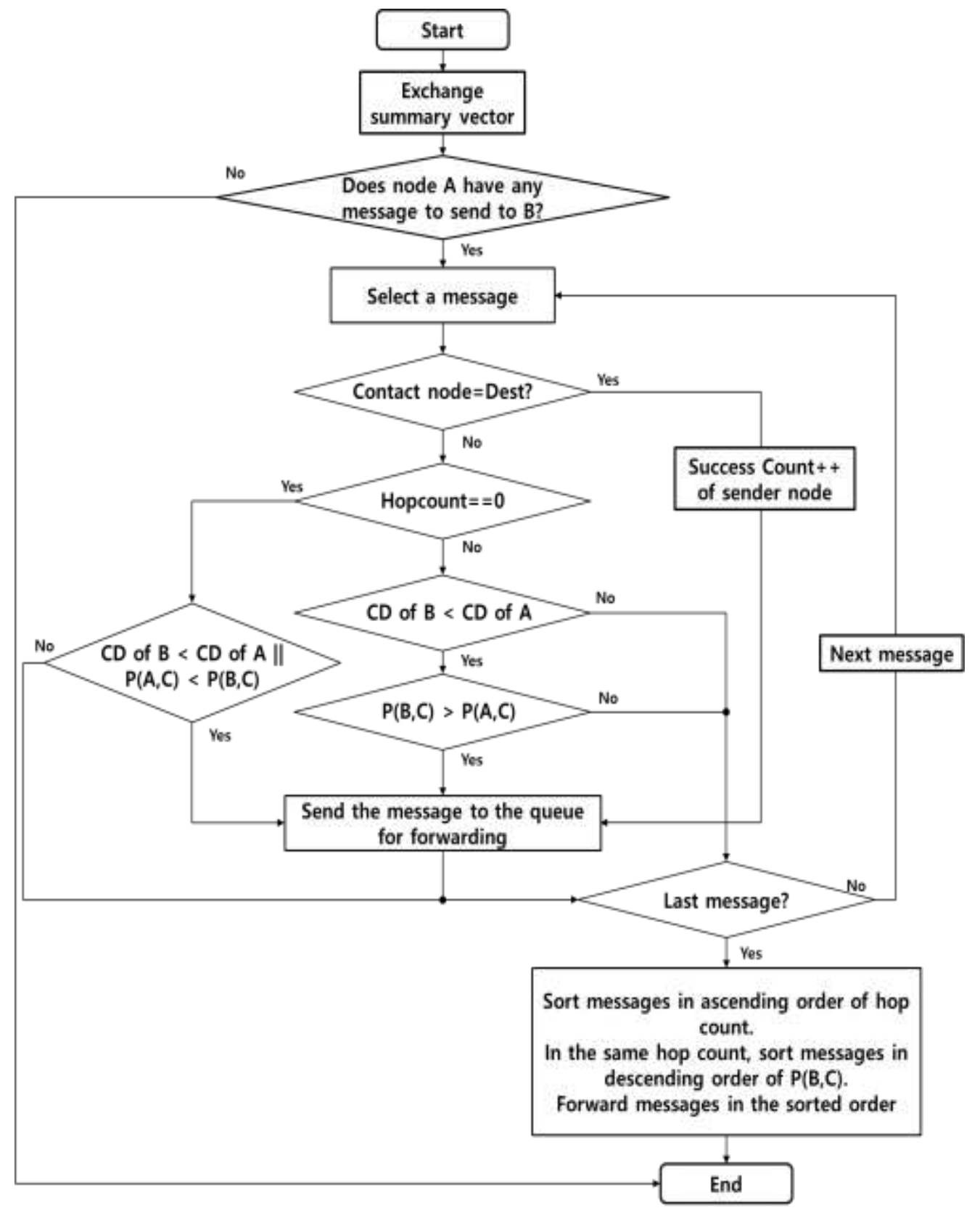

Figure 2. Flowchart of the Proposed Protocol

Figure 3 shows an example scenario of the proposed protocol. Node A's count difference is 17 and node B's count difference is 14 . Since the hop count of message $\mathrm{m} 1$ of node $A$ is 1 , it is selected to be forwarded to node $B$ since $P(B, C)$ is higher than $P(A, C)$. Likewise, messages $\mathrm{m} 3$ and $\mathrm{m} 4$ are selected to be forwarded to node $\mathrm{B}$. Message $\mathrm{m} 2$ is not forwarded to node $B$ since $P(B, D)$ is lower than $P(A, D)$. Although $P(B, G)$ is not higher 
than $P(A, G)$, the message $m 5$ with the destination node $G$ is selected to be forwarded to node $\mathrm{B}$ because hop count of the message $\mathrm{m} 5$ is 0 and thus, needs to be disseminated. The selected messages are sorted on the ascending order of hop count. At node B, the hop counts of the received messages are increased by one.

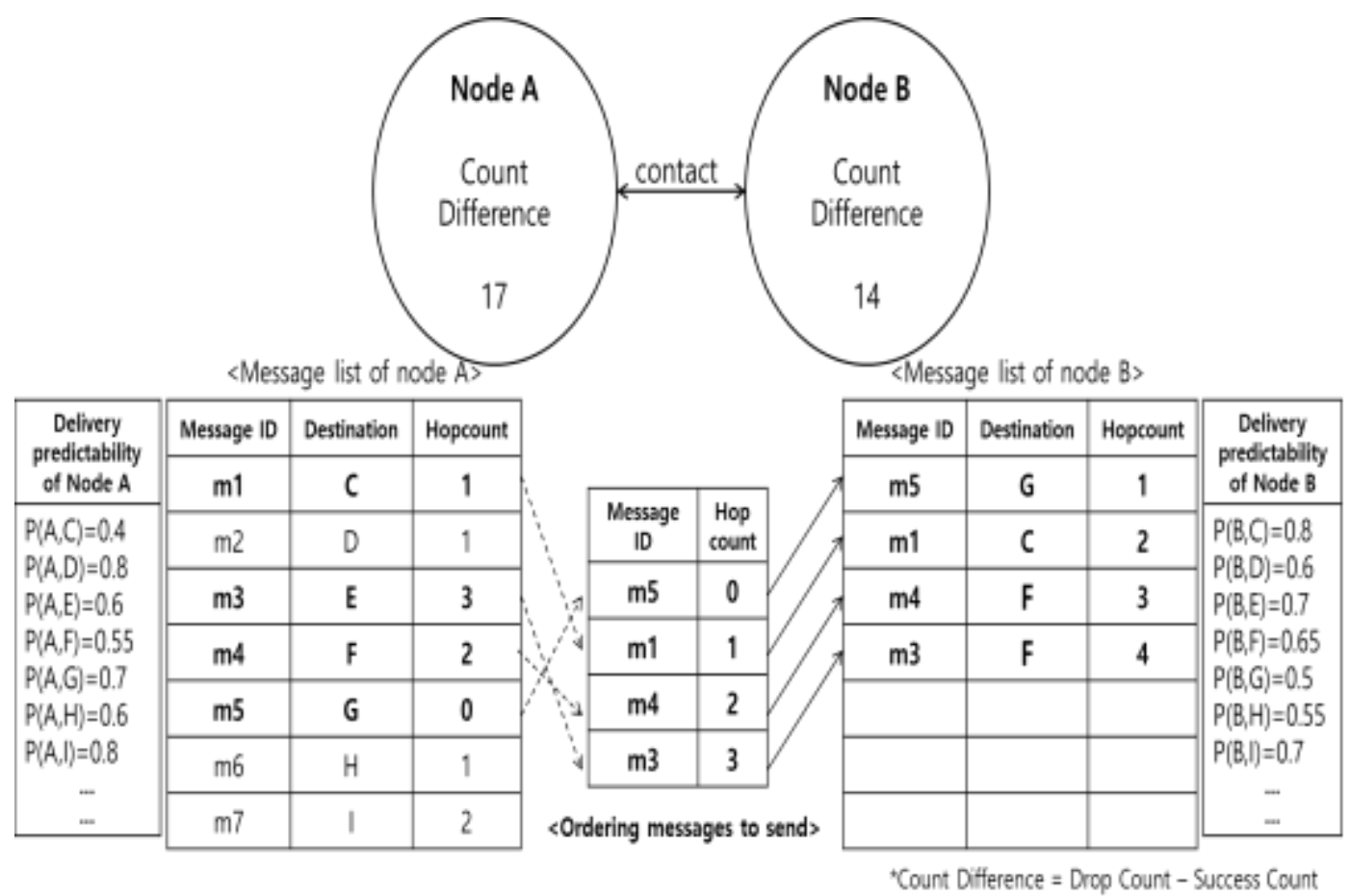

Figure 3. An Example Scenario of the Proposed Protocol

\section{Performance Analysis}

In this paper, the performance of the proposed protocol is analyzed using Opportunistic Network Environment (ONE) simulator developed by Helsinki University [15-16]. The performance of the proposed protocol is compared with that of PRoPHET protocol, from the aspect of delivery ratio, overhead ratio, and delivery latency, which are defined as the following equations $(1)-(3)$ :

$$
\begin{array}{r}
\text { delivery ratio }=\frac{\text { number of delivered messages }}{\text { number of created messages }} \\
\text { overhead ratio }=\frac{\text { number of relayed messages-number of delivered messages }}{\text { number of delivered messages }} \\
\text { delivery latency }=\frac{\text { sum of delivered messages'delay }}{\text { number of delivened messages }}
\end{array}
$$

The values of parameters assumed in this simulation are listed in Table 1. 
Table 1. Simulation Parameters

\begin{tabular}{|l|l|}
\hline \multicolumn{1}{|c|}{ Parameter } & \multicolumn{1}{c|}{ Value } \\
\hline Router & PRoPHET Router \\
\hline Movement model & $\begin{array}{l}\text { pedestrians, car: Shortest Path Map Based } \\
\text { tram: Map Route Movement }\end{array}$ \\
\hline Number of Node & 126 (pedestrians= car=, tram=6) \\
\hline Simulation Time & $43,200 \mathrm{~s}$ \\
\hline Transmission range(m) & 10 \\
\hline Packet transmission speed & $250 \mathrm{Kbyte} / \mathrm{s}$ \\
\hline Buffer Size(Bytes) & $10 \sim 100 \mathrm{M}$ \\
\hline Message interval(s) & $25 \sim 35$ \\
\hline Message size(Bytes) & $500 \mathrm{k} \sim 1 \mathrm{M}$ \\
\hline
\end{tabular}

Figures 4 6 show delivery ratio, overhead ratio, and delivery latency of Proposed(Proposed_sort), Proposed(GRTRMax_sort), and PRoPHET(GRTRMax_sort) [4], for varying the buffer size. In Proposed(Proposed_sort) protocol, node sorts messages in the ascending order of hop count firstly and transmitted based on the sorted order. If there are messages with the same hop count, then the messages are sorted based on the descending order of delivery predictability of the receiving node. In Proposed(GRTRMax_sort) protocol, messages are selected based on the proposed selection scheme and sorted in the descending order of delivery predictability of the receiving node, where sorting strategy of GRTRMax was originally proposed in [4]. In PRoPHET(GRTRMax_sort) protocol, messages are selected if the delivery predictability of the receiving node is higher than that of the sending node and sorted in the descending order of delivery predictability of the receiving node.

As Figure 4 shows, the delivery ratios of all the protocols increase as buffer size increases since higher buffer size accommodate more messages and reduce message drop. The delivery ratios of the proposed protocols are higher than PRoPHET since the proposed protocol can remove unnecessarily forwarding by using count difference and thus delivery ratio can increase. The proposed protocol with the proposed sorting strategy has the largest delivery ratio.

Figure 5 shows overhead ratio for varying the buffer size. The delivery ratios of the proposed protocols are smaller than PRoPHET since the proposed protocol can remove unnecessarily forwarding by using count difference. The overhead ratio of the proposed protocols increases as buffer size increase, because the effect of sharply increased relayed messages is more dominant than the increased delivered messages. However, the overhead ratio of the PRoPHET protocol decreases as buffer size increase, because the effect of sharply increased delivered messages is more dominant that the increased relayed messages. The proposed protocol with the proposed sorting strategy has the smallest delivery ratio.

Figure 6 shows delivery latency for varying the buffer size. The delivery latencies of the proposed protocols are smaller than PRoPHET since the proposed protocol can remove unnecessarily forwarding by using count difference, and thus, message can be delivered to a destination node more rapidly. The proposed protocol with the proposed sorting strategy has the smallest delivery latency. 


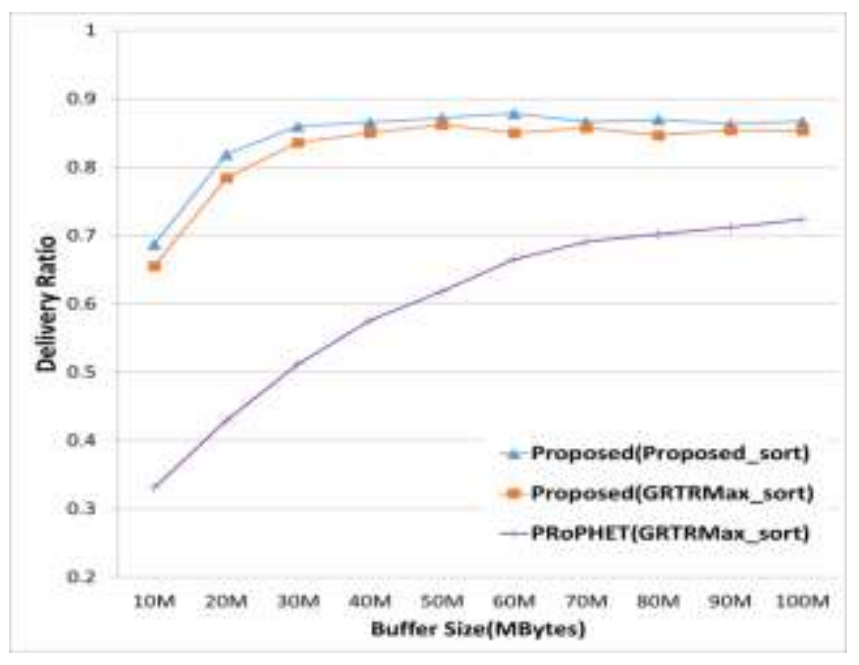

Figure 4. Delivery Ratio for Varying Buffer Size

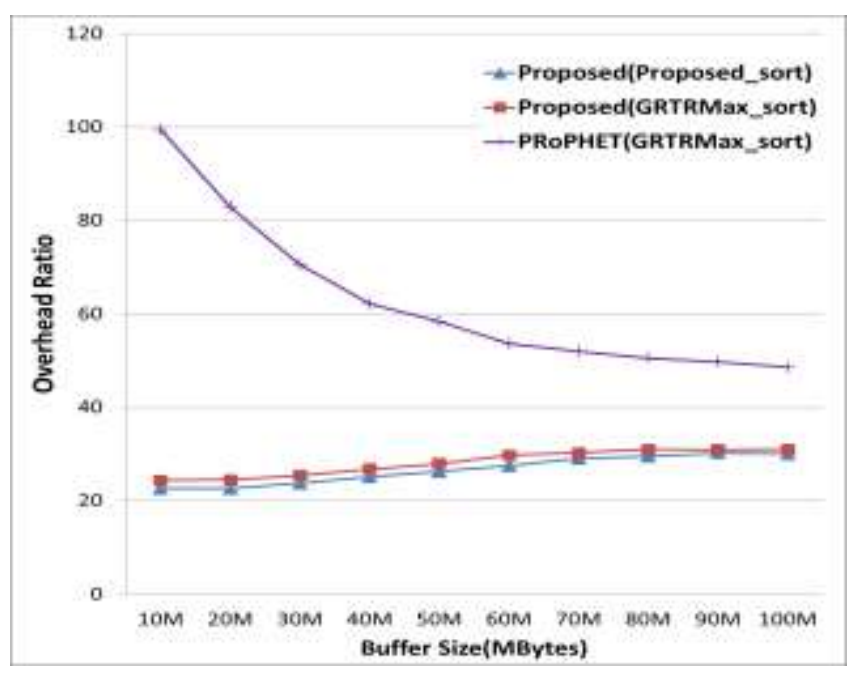

Figure 5. Overhead Ratio for Varying Buffer Size

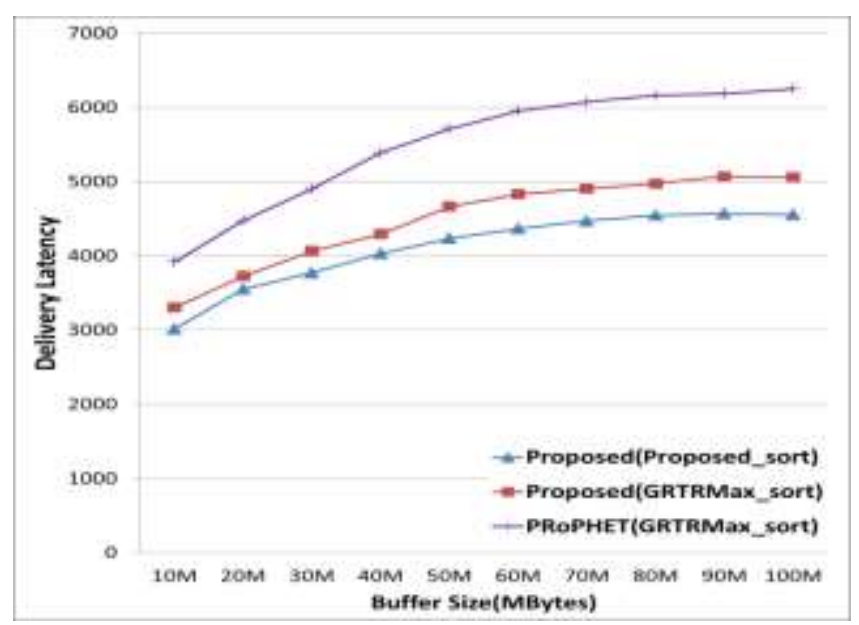

Figure 6. Delivery Latency for Varying Buffer Size

Figures 7 9 show delivery ratio, overhead ratio, and delivery latency of Proposed(Proposed_sort), Proposed(GRTRMax_sort), and PRoPHET(GRTRMax_sort) [4], for varying message interval. As Figure 7 shows, the delivery ratios of all the 
protocols increase as message interval increases since higher message interval generates less messages and thus, a node with a fixed buffer size accommodates more messages and message drop is reduced. The delivery ratios of the proposed protocols are higher than PRoPHET and the proposed protocol with the proposed sorting strategy has the largest delivery ratio.

Figure 8 shows overhead ratio for varying the message interval. The overhead ratios of all the protocols increase as message interval increases. This is because the effect of sharply increased relayed messages is more dominant than the increased delivered messages. The overhead ratios of the proposed protocols are smaller than PRoPHET and the proposed protocol with the proposed sorting strategy has the smallest overhead ratio.

Figure 10 shows delivery latency for varying the message interval. The delivery latencies of the proposed protocols are smaller than PRoPHET since the proposed protocol can remove unnecessarily forwarding by using count difference, and thus, message can be delivered to a destination node more rapidly. The proposed protocol with the proposed sorting strategy has the smallest delivery latency.

Figures 10 12 show delivery ratio, overhead ratio, and delivery latency of Proposed(Proposed_sort), Proposed(GRTRMax_sort), and PRoPHET(GRTRMax_sort) [4], for varying number of nodes. As Figure 10 shows, the delivery ratios of the proposed protocols increase due to more dissemination of messages. However, the delivery ratio of the PRoPHET protocol decreases as the number of nodes increases for large values of the number of nodes. This is because more dissemination of messages in PRoPHET results in more message drop and degrade delivery ratio. The proposed protocol with the proposed sorting strategy has the highest delivery ratio.

Figure 11 shows overhead ratio for varying the message interval. The overhead ratios of all the protocols increase as message interval increases. This is because the effect of sharply increased relayed messages is more dominant than the increased delivered messages, especially for PRoPHET protocol. The overhead ratios of the proposed protocols are smaller than PRoPHET and the proposed protocol with the proposed sorting strategy has the smallest overhead ratio.

Figure 12 shows delivery latency for varying the message interval. The delivery latencies of the proposed protocols are smaller than PRoPHET since the proposed protocol can remove unnecessarily forwarding by using count difference, and thus, message can be delivered to a destination node more rapidly. The proposed protocol with the proposed sorting strategy has the smallest delivery latency.

\section{Summary}

In this paper, a novel routing protocol using contact information on successful message delivery and buffer overflow was proposed in opportunistic delay tolerant networks, where a message is forwarded to another node with lower count difference as well as higher delivery predictability for the considered message. An example scenario of the proposed protocol was illustrated and a detailed algorithm of the proposed protocol was presented using a flowchart. Then, the performance of the proposed protocol was analyzed in detail, from the aspect of delivery ratio, overhead ratio, and delivery latency, by varying the buffer size, message interval, and number of nodes. Numerical results show that the proposed protocols have better delivery ratio, overhead ratio, and delivery latency by efficiently removing unnecessary forwarding by using count difference. Especially, the proposed protocol with the proposed sorting strategy has the best performance always. 


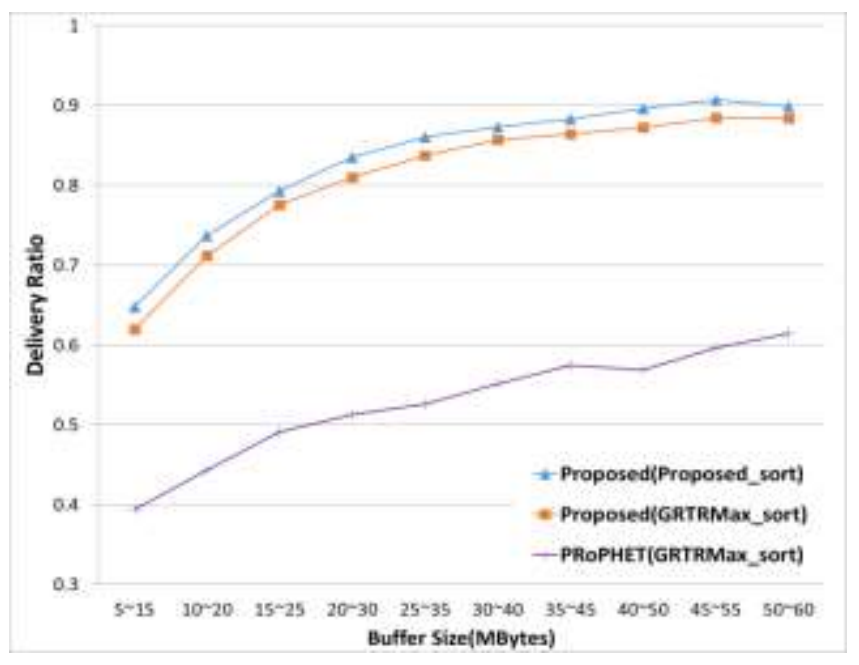

Figure 7. Delivery Ratio for Varying Message Interval

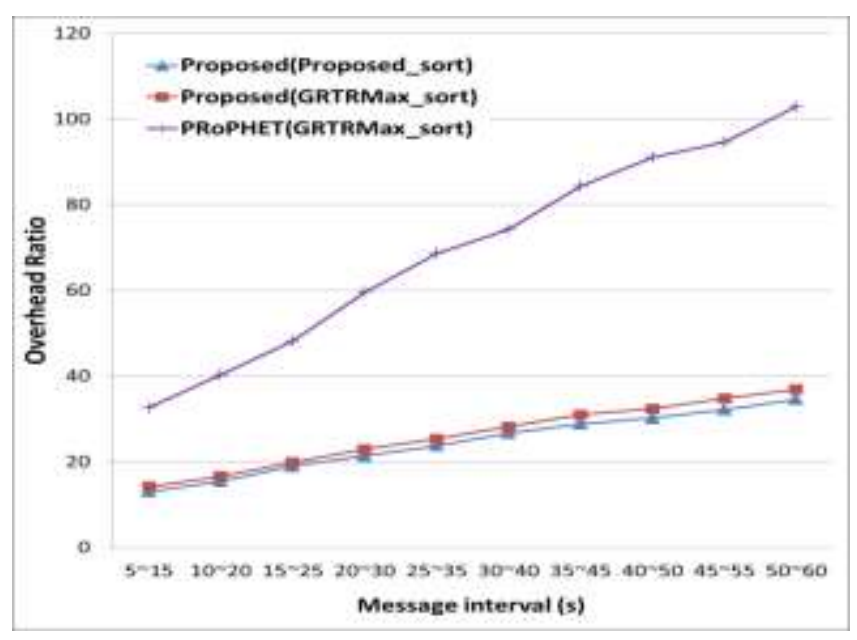

Figure 8. Overhead Ratio for Varying Message Interval

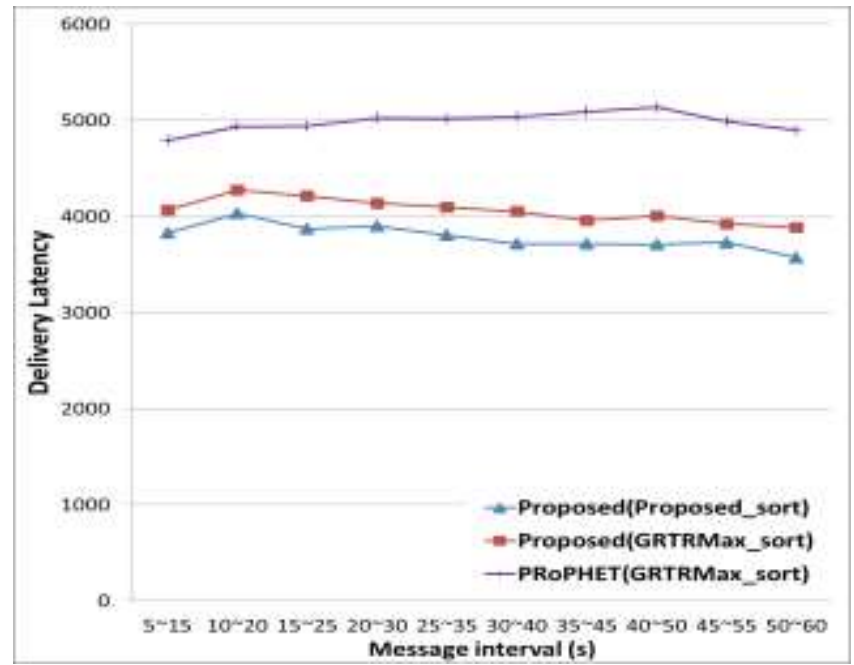

Figure 9. Delivery Latency for Varying Message Interval 


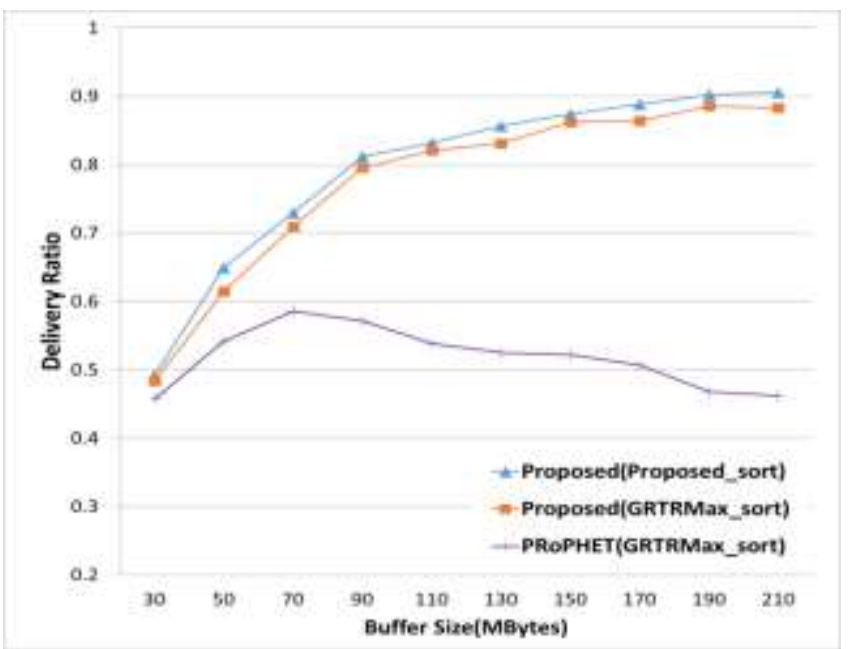

Figure 10. Delivery Ratio for Varying Number of Nodes

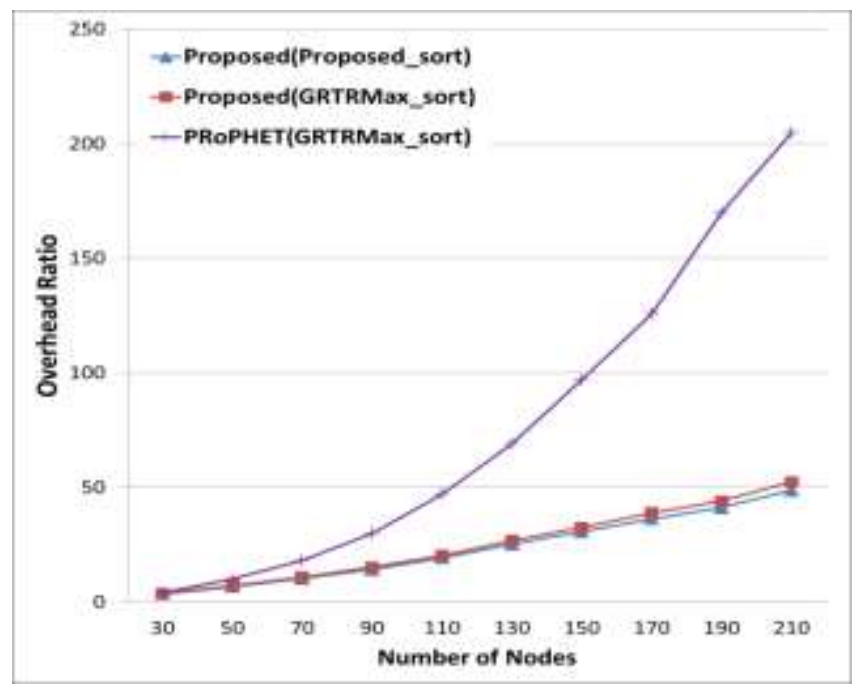

Figure 11. Overhead Ratio for Varying Number of Nodes

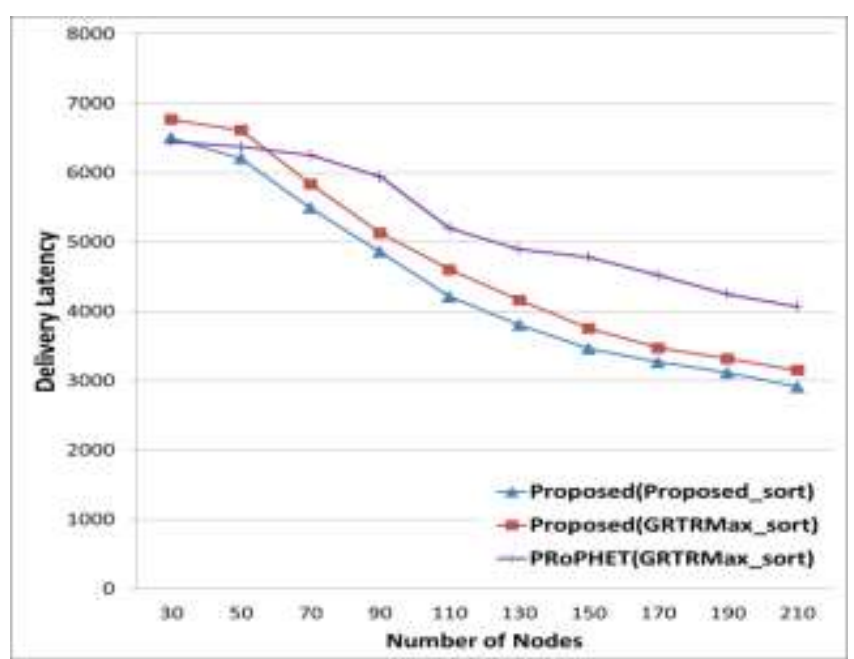

Figure 12. Delivery Latency for Varying Number of Nodes 


\section{Acknowledgments}

This research of Dong Young Seo and Yun Won Chung was supported in part by Basic Science Research Program through the National Research Foundation of Korea (NRF) funded by the Ministry of Science, ICT \& Future Planning (NRF-2014R1A1A1037728). This paper is a revised and expanded version of a paper entitled "An Improved Opportunistic Routing Protocol using Message Drop Count and Success Count" presented at 9th International Conference on Grid and Distributed Computing, Jeju Island, Korea on 21 23 December, 2016.

\section{References}

[1] Delay Tolerant Networking Research Group, http://www.dtnrg.org.

[2] Y. Cao, and Z. Sun, "Routing in Delay/Disruption Tolerant Networks: A Taxonomy, Survey and Challenges", IEEE Communications Surveys \& Tutorials, vol. 15, (2013), pp.654-677.

[3] Z. Zhang, "Routing in intermittently connected mobile ad hoc networks and delay tolerant networks: overview and challenges", IEEE Communications Survey and Tutorial, vol. 8, (2006), pp. 24-37

[4] A. Lindgren, A. Doria, E. Davies, and S. Grasic, "Probabilistic routing protocol for intermittently connected networks", IETF RFC 6693, (2012).

[5] X. Zhang, G. Neglia, J. Kurose, and D. Towsley, "Performance modeling of epidemic routing", Computer Networks, vol. 51, no. 10, (2007), pp. 2867-2891.

[6] K. H. Lee, T. M. Park, and C. K. Kim, "Social-community based DTN Routing. Journal of Korean Institute of Information Scientists and Engineers", vol. 38, (2011), pp. 366-373.

[7] Y. Liu, J. Wang, S. Zhang, and H. Zhou, "A Buffer Management Scheme Base on Message Transmission Status in Delay Tolerant Network", Proceedings of IEEE Wireless Communications and Networking Conference, (2011).

[8] M. J. Kim, and Y. W. Chung, "An Improved Message Delivery Scheme Based on Node Density in Delay Tolerant Network", Journal of Korean Institute of Information Technology, vol. 12, (2014), pp. 69-74.

[9] H. J. Lee, J. C. Nam, W. K. Seo, J. I. Choi, and Y. Z. Cho, “An Efficient DTN Routing Protocol with Considering Contact Duration”, Journal of Korean Institute of Communication Sciences, (2015), pp. 921-922.

[10] T. K. Huang, C. K. Lee, and L. J. Chen, "PRoPHET+: anadaptive PRoPHET-based routing protocol for opportunistic network," in Proceedings of the 24th IEEE International Conference on Advanced Information Networking and Applications, (2010), pp. 112-119.

[11] A. E. Ouadrhiri, M. E. Kamili, M. R. E. Fenni, and L. Omari, "Learning controlled forwarding strategy improving probabilistic routing in DTNs", Proceedings of IEEE Wireless Communications and Networking Conference, (2014).

[12] P. Wang, and F. Shen, "Method to improve the performance of PROPHET routing protocol", Proceedings of the 2nd IEEE International Workshop on Education Technology and Computer Science, (2010), pp. 248-251.

[13] S. H. Park, D. Y. Seo, and Y. W. Chung, "An Improved PRoPHET Protocol Using Message Delivery Count to Destination Nodes", Journal of Korean Institute of Information Technology, vol. 14, (2016), pp. $75-80$.

[14] S. W. Kim, D. Y. Seo, and Y. W. Chung, "An Improved Opportunistic Routing Protocol using Message Drop Count and Success Count," Proceedings of Grid and Distributed Computing, (2016).

[15] The Opportunistic Network Environment simulation, http://www.netlab.tkk.fi/tutkimus/ dtn/theone/

[16] A. Keranen, J. Ott, and T. Karkkainen "The ONE simulator for DTN protocol evaluation", Proceedings of the second International Conference on Simulation Tools and Techniques, (2009), pp. 55:1-10

\section{Authors}

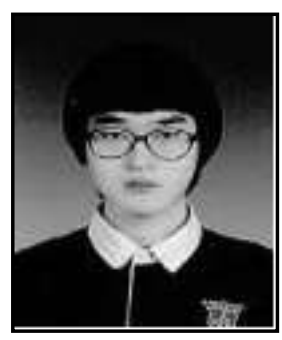

Sun Woong Kim, He is an undergraduate student of school of electronic engineering of Soongsil University, Seoul, Korea. His research interests include opportunistic network and delay tolerant networks. 


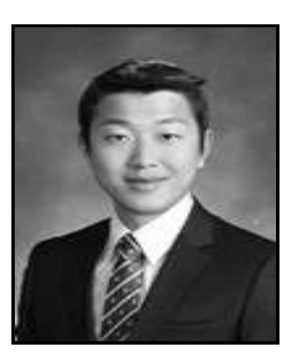

Dong Young Seo, He received his B.S. degree in Electronic Engineering of Soongsil University in 2015. He is currently an MS student in the department of Information and Communication in Soongsil University. His research interests include delay tolerant network, green cellular networks, LTE, and 5G networks.

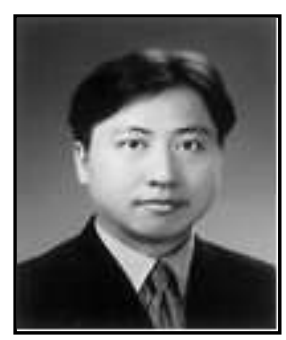

Yun Won Chung, He received his B.S., M.S., and Ph.D. degrees from school of electrical engineering of Korea Advanced Institute of Science and Technology (KAIST), Deajeon, Korea, in 1995, 1997, and 2001, respectively. He was a visiting post-doctoral researcher at the Centre for Telecommunications Research of King's College London, London, UK from 2001 to 2002. He was a research staff at Electronics and Telecommunications Research Institute (ETRI) and developed WiBro system based on IEEE 802.16e and 802.16g standards. Currently, he is an Associate Professor of school of electronic engineering of Soongsil University. His research interests include modelling and performance analysis of various wireless networks such as cellular networks including 3G, 4G LET, and future SDN/NFV-based 5G, mobile ad hoc networks (MANET), vehicular ad hoc networks (VANET), wireless sensor networks (WSN), and opportunistic delay tolerant networks (DTN). Green network technology is another research interest, too. 